\title{
Change of the duodenal mucosa-associated microbiota is related to intestinal metaplasia
}

\author{
Jian Gong ${ }^{1,2}$, Lixiang $\mathrm{Li}^{1,3}$, Xiuli Zuo ${ }^{1,3}$ and Yanqing $\mathrm{Li}^{1,3^{*}}$
}

\begin{abstract}
Background: In this study, we aimed to investigate the characteristics of the duodenal mucosal microbiota of patients with intestinal metaplasia (IM) and compare it with those of the gastric mucosal microbiota.

Method: We collected the duodenal and gastric mucosal samples from 10 adult patients with IM and 10 healthy controls (HC). The V3-V4 region of the bacterial $16 \mathrm{~S}$ rRNA gene was examined by high throughput sequencing method.

Results: The diversity of the HC duodenal microbiota was higher than that of IM patient based on the Shannon and Simpson index while the Chao indices of IM duodenal mucosal microbiota was significantly higher than that of gastric mucosal microbiota of patients with IM. There was a marked difference in the duodenal microbiota structure between patients with IM and HC (ANOSIM, R=1, $P=0.001$ ). We also found that the Helicobacter pylori infection in gastric mucosa did not influence the structure of duodenal mucosal microbiota. The gastric mucosal microbiota structure significantly differed between patients with IM and $\mathrm{HC}$ who were $\mathrm{H}$. pylori-negative (ANOSIM, R $=0.452$, $P=0.042$ ) or $\mathrm{H}$. pylori-positive (ANOSIM, $R=0.548, P=0.003$ ), respectively. For duodenal mucosal microbiota, genera Lactococcus, Flavobacterium, Psychrobacter, Mysroides, Enhydrobacter, Streptococcus, and Leuconostoc were enriched in patients with IM. In contrast, genera Bacillus, Solibacillus, Lysinibacillus, Exiguobacterium, Oceanobacillus, and Paenibacillus were enriched in $\mathrm{HC}$.
\end{abstract}

Conclusion: A marked dysbiosis duodenal mucosal microbiota in patients with IM was observed, and this dysbiosis might be responsible for IM pathogenesis.

Keywords: Intestinal metaplasia, Duodenal microbiota, Dysbiosis, Helicobacter pylori

\section{Background}

Recently, gastric cancer (GC) has been reported as the fourth most common malignancy and one of the leading causes of cancer-related deaths worldwide. It has a particularly high incidence in East Asia, Eastern Europe, and Central and South America [1]. Gastric carcinogenesis has been hypothesized as a multistep process comprising superficial gastritis (SG), chronic gastritis, atrophic gastritis (AG), intestinal metaplasia (IM), dysplasia, and then carcinoma [2]. IM is a crucial risk factor for GC and is considered part of the pathologic

\footnotetext{
* Correspondence: liyanqing@sdu.edu.cn

'Department of Gastroenterology, Qilu Hospital, Shandong University, Jinan 250012, China

${ }^{3}$ Laboratory of Translational Gastroenterology, Qilu Hospital, Shandong

University, No. 107, Wenhuaxi Road, Jinan 250012, China

Full list of author information is available at the end of the article
}

spectrum of gastric mucosal atrophy [3]. According to epidemiological evidence, IM condition may be reversed following treatment with antioxidant agents for eradicating Helicobacter pylori [4]. However, IM is still believed to be the "point of no return" during the histological process ranging from chronic gastritis to cancer [5]. Thus, it is crucial to explore the molecular mechanisms underlying IM pathogenesis and develop strategies to interfere with the gastric carcinogenesis.

Recent studies show that microbial changes are related to the histological stages of gastric tumorigenesis. Chronic $H$. pylori infection can cause mucosal inflammation and induce histological change. It is also recognized as a major risk factor for GC. Nevertheless, only $3 \%$ of $\mathrm{H}$. pylori-infected patients develop GC [6]. Moreover, it was found

(C) The Author(s). 2019 Open Access This article is distributed under the terms of the Creative Commons Attribution 4.0 International License (http://creativecommons.org/licenses/by/4.0/), which permits unrestricted use, distribution, and 
that $H$. pylori is usually undetectable in gastric cancer samples [7]. These studies suggest that $H$. pylori infection might only be an early event for the gastric mucosa which would further undergo oncogenic changes, and indicate the potential role of mucosal microbes, with the exception of $\mathrm{H}$. pylori, in gastric carcinogenesis. The dominant phylum in mucosal microbes was Proteobacteria in both H. pylori-negative and H. pylori-positive samples [8]. Two previous studies demonstrated that the microbiota of patients with IM was found to partially overlap with the gastritis and cancer group among patients with $H$. pylori infection $[9,10]$. Li et al. (2017) found that the microbiota of gastritis samples mostly overlapped with that of IM samples. In contrast, microbiota of patients with IM and GC had significantly low microbial richness, while the $\beta$ diversity of microbiota of SG, AG and IM was similar in overall differences, with the exception of that of GC [11]. These conflicting results suggest that IM might be the key point in microbiota change and there might be other potential factors involved in gastric tumorigenesis, especially in patients with IM.

Most of the studies on gastric cancer have focused on gastric microbiota dysbiosis. Recent evidence has revealed that the small intestinal microbiota, especially the mucosal microbiota, might play a crucial role in gastrointestinal health [12]. Dysbiosis of the small intestinal microbiota has been found in celiac disease [13], chronic liver disease [14], diabetes mellitus [15], and irritable bowel syndrome [16]. However, the information regarding the role of duodenal microbiota in IM is still limited.

In this study, we investigated the mucosal microbiota of the duodenum and stomach in patients with IM and compared it with those of $\mathrm{HC}$.

\section{Results \\ Participants}

A total of 20 participants, including 10 IM (6 males, 4 females, 6 HP-positive) and 10 healthy individuals as control (5 males, 5 females, 4 HP-positive) were recruited in this study (Table 1 and Additional file 1: Table S1). No significant differences in gender (male: $60.0 \%$ vs. $50.0 \%, P=$ $0.65)$ and age $(51.3 \pm 8.01$ vs. $57.80 \pm 7.22, P=0.07)$ were detected between the IM and $\mathrm{HC}$ groups, respectively.

Table 1 Characteristics of the study participants

\begin{tabular}{llll}
\hline & IM $(n=10)$ & $H C(n=10)$ & $P$ \\
\hline Sex (male, \%) & $6(60 \%)$ & $5(50 \%)$ & 0.65 \\
Age & $57.8 \pm 7.22$ & $51.30 \pm 8.01$ & 0.31 \\
HP+ (16 s RNA sequencing) & 6 & 4 & 0.37 \\
HP+ (RUT) & 6 & 4 & 0.37 \\
\hline RUT rapid biopsy urease test & & &
\end{tabular}

RUT rapid biopsy urease test

\section{Small intestinal bacterial diversity is lower in patients} with IM

To detect the microbiota dysbiosis associated with IM, the microbial diversity and richness of gastric and duodenal mucosal biopsy samples were estimated by analyzing of hypervariable $\mathrm{V} 3-\mathrm{V} 4$ regions of the $16 \mathrm{~S}$ ribosomal RNA gene. An average of 37,165 high quality sequences per sample was obtained after quality-filtering steps. The estimate of coverage reached $>99.9 \%$ for all samples. After removing the rare microbial OTUs, 27,698 sequences per sample and 125 OTUs were obtained for further analysis. Next, we estimated the $\alpha$-diversity of the microbiota (Additional file 1: Table S1) and compared the mean values between groups. The results are shown in Fig. 1. Compared with IMG and IMD, microbiota of HC-G and HC-D had slightly reduced Chaol-estimated microbial richness with no statistical differences (data not shown). However, the diversity of HC-D was higher than that of IM-D based on the Shannon and Simpson indices. Meanwhile, the two Chao indices of duodenal mucosal microbiota were higher than those of gastric mucosa and only the Chao index of IM duodenal mucosal microbiota was significantly higher than that of IM-G (Wilcoxon rank-sum test, $P<0.05$ ).

\section{Gastric and duodenal microbiota structure is altered in patients with IM}

The similarity of the bacterial community structures between patients with IM and HC was evaluated by PCoA (Fig. 2a). For duodenal mucosal microbiota, significant differences were observed in the microbiota structure between IMD and HC-D (ANOSIM, R $=1, P=0.001$ ). We also found that the HP infection in gastric mucosa did not influence the structure of duodenal mucosa microbiota (Fig. 2b). The gastric mucosal microbiota structure significantly differed between IMG and HC-G in HP-negative patients (ANOSIM, $\mathrm{R}=0.452, P=0.042$ ) or HP-positive patients (ANOSIM, $\mathrm{R}=0.548, P=0.003$ ), respectively. There was no significant difference between IMG (HP-) and IMD (ANOSIM, $\mathrm{R}=0.37, P=0.05$ ), as well as between HC-G (HP-) and HC-D (ANOSIM, R = $0.176, P=0.075)$.

\section{Duodenal mucosa microbiota composition is altered in patients with IM}

Most of the gastric and duodenal mucosal bacteria detected in this study belong to the following three phyla: Firmicutes, Proteobacteria, and Actinobacteria (Fig. 3a). The main nine genera of gut microbiota (percentages were above $1 \%$ ) comprised up to $90 \%$ of the total microbiota and included the following: Lactococcus, Bacillus, Helicobacter, Solibacillus, Pseudomonas, Arthrobacter, Lysinibacillus, and Streptococcus (Fig. 3b). We compared the proportions of dominant genera and found that most 

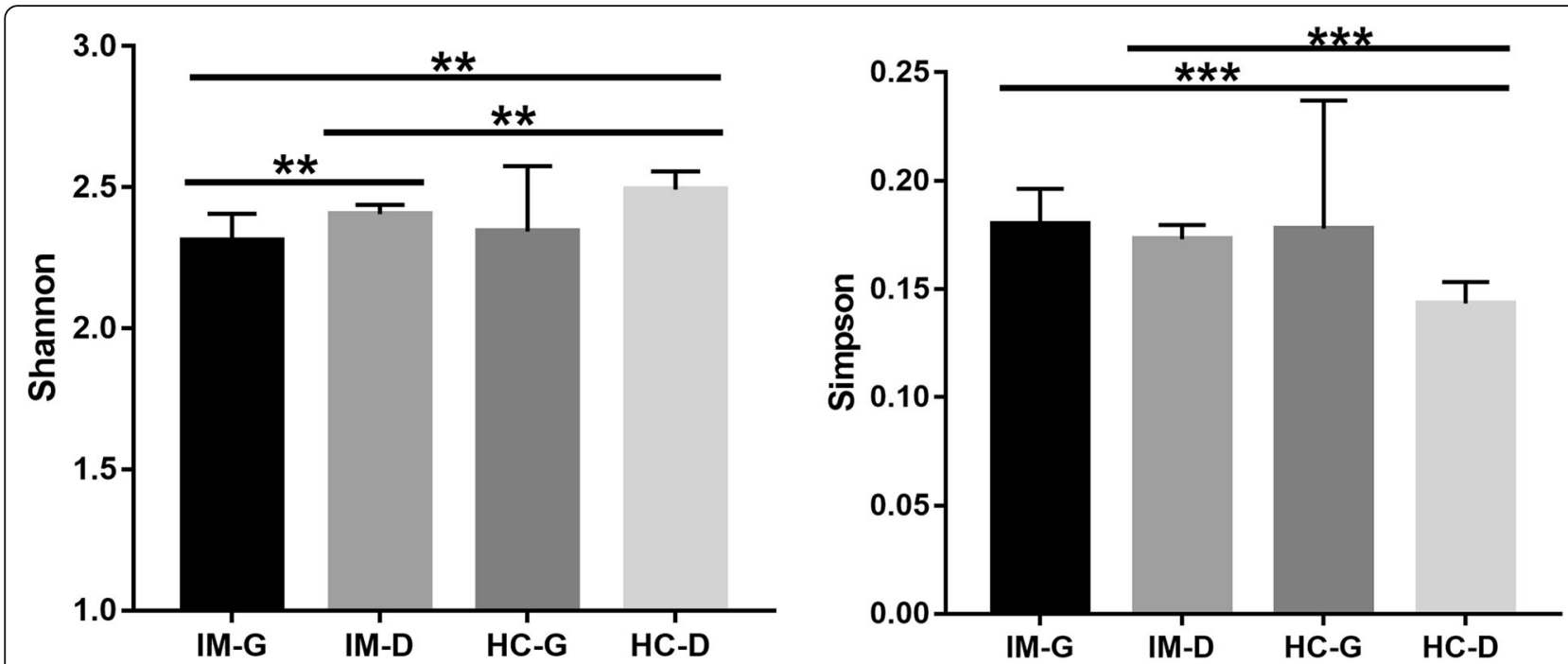

Fig. 1 The a-diversity of the gut microbiota in HSP and control. Unpaired t-test were used for comparing the Ace and Shannon index. ${ }^{*}, P<0.05$; ${ }^{* *}, P<0.01,{ }^{* * *}, P<0.001$

of them changed as shown in Fig. 3c. The specific taxa that most likely contributed to the differences between IM and $\mathrm{HC}$ group were revealed by linear discriminant analysis of effect size (Fig. 4). In duodenal mucosal microbiota, the genera Lactococcus, Flavobacterium, Psychrobacter, Mysroides, Enhydrobacter, Streptococcus, and Leuconostoc was found to be enriched in patients with IM. In contrast, the genera Bacillus, Solibacillus, Lysinibacillus, Exiguobacterium, Oceanobacillus, and Paenibacillus were enriched in $\mathrm{HC}$ (Fig. 4a). However, there were no significant specific taxa in gastric mucosa microbiota between IM and HC (data not shown). There was also a greater number of specific taxa between gastric and duodenal mucosa microbiota in patients with IM than HC. Eighteen genera including Bacillus, Solibacillus, and Arthrobacter were enhanced in duodenal mucosal microbiota of patients with IM and only three genera, including Variovorax, Acinetobacter, and Oceanobacillus, were enhanced in the duodenal mucosa microbiota of HC (Fig. 4c and d). When the microbiota of four groups was compared, four genera including Flavobacterium Enhydrobacter, Psychrobacter, and Streptococcus were found to be enriched in the duodenal mucosal microbiota of patients with IM, while the genera Bacillus, Oceanobacillus, Solibacillus, and Exiguobacterium were enriched in duodenal mucosal microbiota
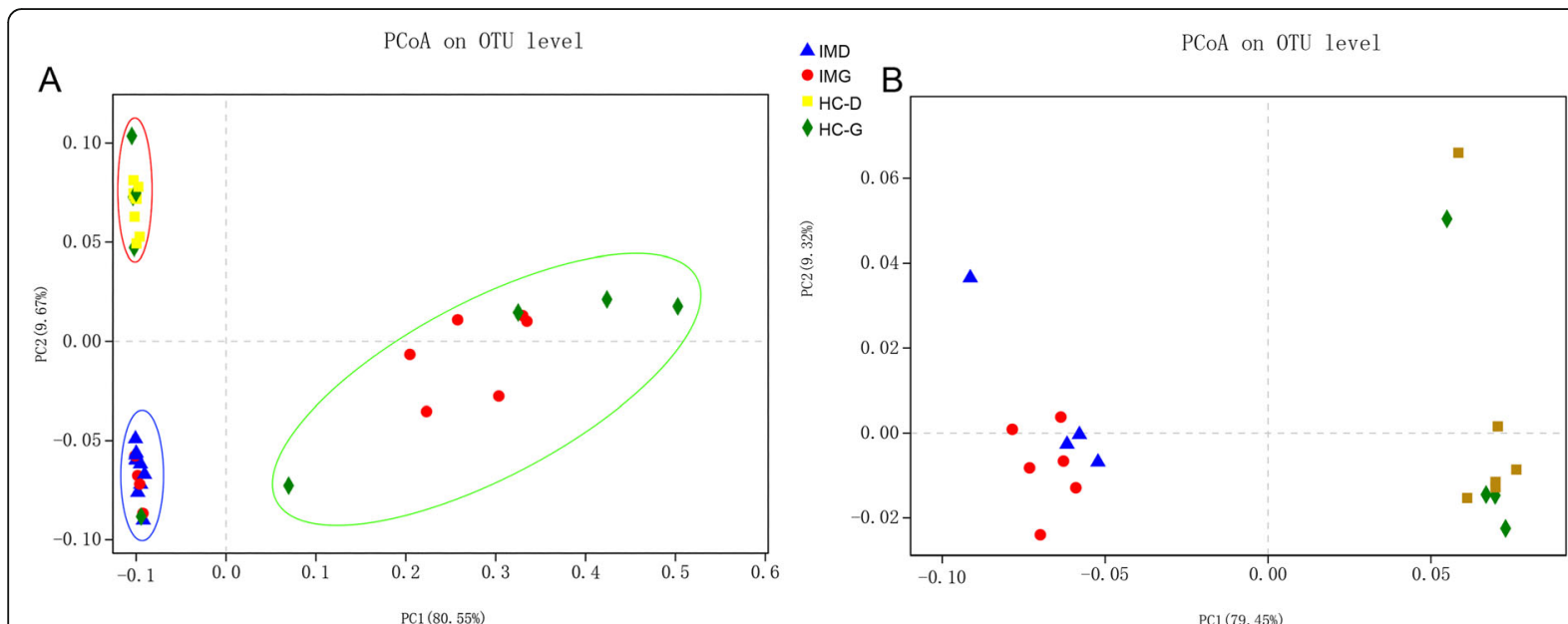

Fig. 2 PCOA analysis of the microbiota among IM and HC. a, Comparison of the duodenal and gastric microbiota. Green circle, gastric samples with HP infection; red circle, duodenal and gastric sample of HC; blue circle, duodenal and gastric sample of IM. HCD, duodenal samples of HC; HCG, gastric sample of HC; IMG, gastric sample of HC; IMD, duodenal samples of IM. $\mathbf{b}$, The influence of HP infection in duodenal microbiota. IMD duodenal samples of IM without HP infection; IMDHP, duodenal samples of IM with HP infection; HC, duodenal samples of HC without HP infection; HCHP, duodenal samples of HC with HP infection 


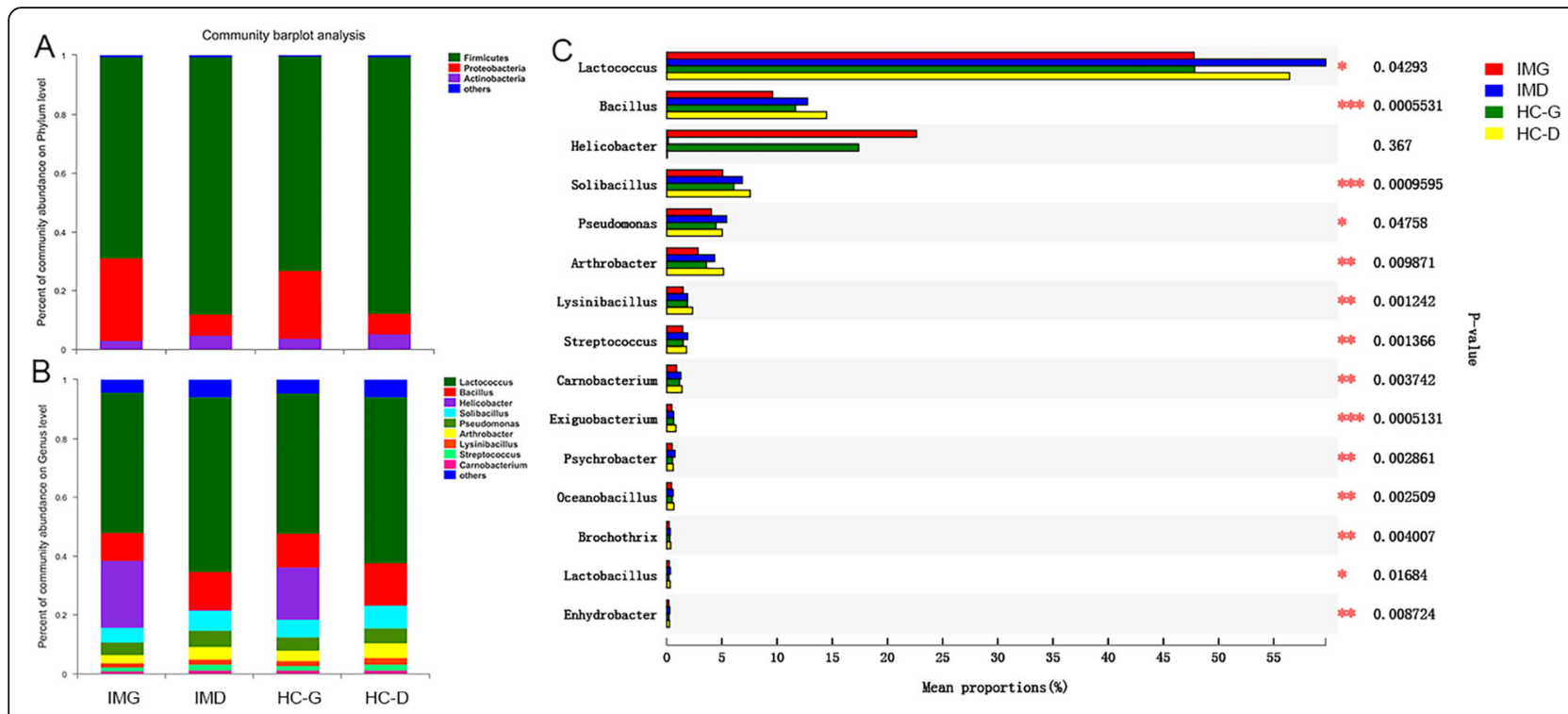

Fig. 3 The relative taxa abundance between IM and HC. a, relative taxa abundance in phylum level; $\mathbf{b}$, relative taxa abundance genus level; $\mathbf{c}$, comparison of relative taxa abundance of genus level

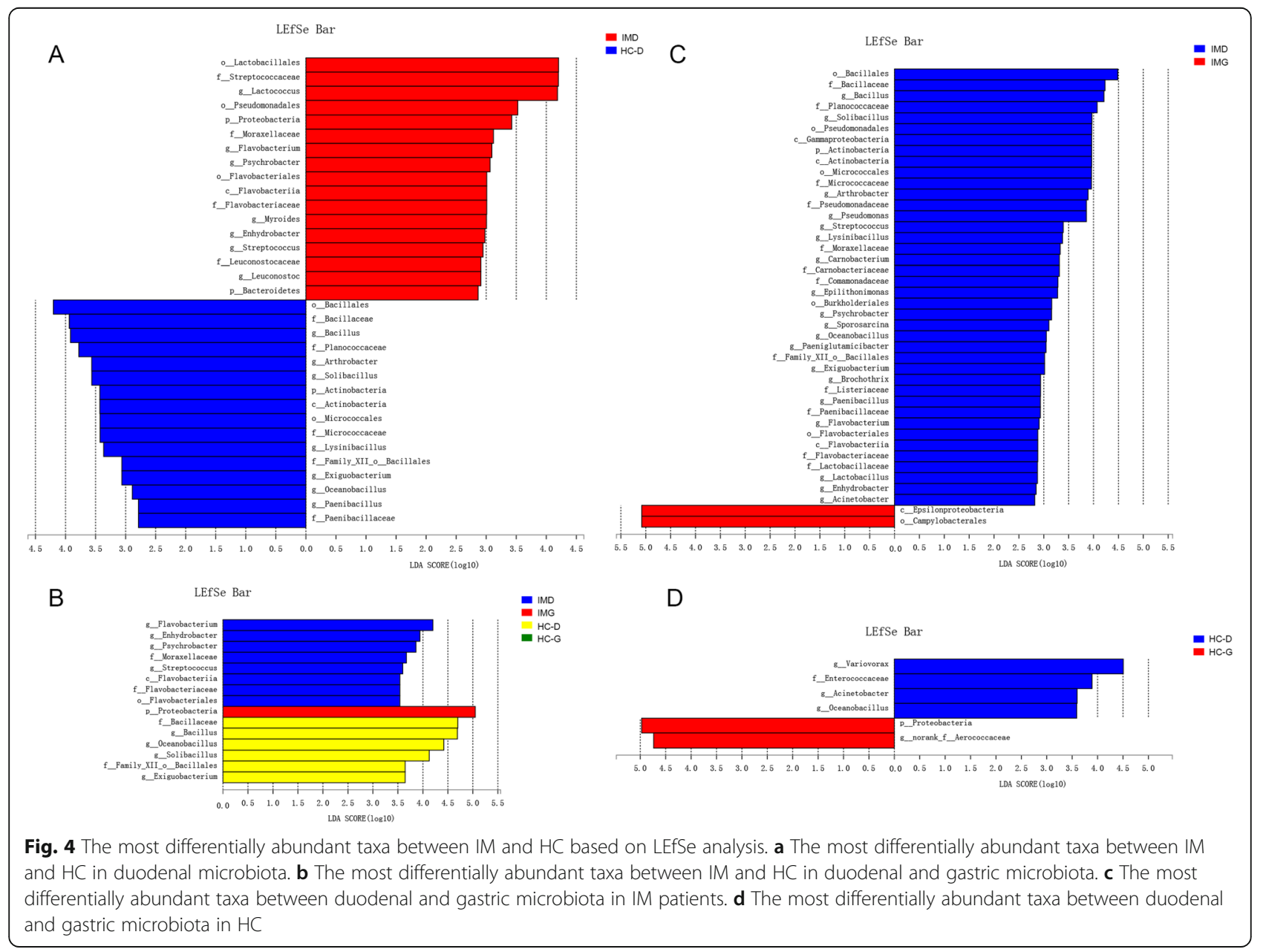


of $\mathrm{HC}$ (Fig. 4b). There was no specific genus enriched in the gastric mucosa microbiota of patients with IM and HC.

\section{Discussion}

In this study, microbial communities in the duodenal mucosa of patients with IM showed significant differences with those of $\mathrm{HC}$, including a lower diversity, different microbiota structure and specific taxa. We also found that the gastric mucosal microbiota of $\mathrm{HC}$ was similar to their duodenal mucosal microbiota. In contrast, the gastric mucosal microbiota of patients with IM differed from their duodenal mucosal microbiota. These data indicated a potential role for duodenum microbiota in IM pathology.

Microbiota dysbiosis has been detected in many gastrointestinal and systemic diseases including IM and gastric cancers. However, the changes in gastric microbiome compositions including microbial diversity and richness across stages of gastric carcinogenesis are inconsistent in different studies [8-11]. It has been previously reported that the diversity, evenness and overall composition was similar between patients with nonatrophic gastritis and patients with IM [7, 9, 10] [7]. In contrast, Li et al. (2017) found that the normal group had higher Shannon and phylogenetic diversity indices than those of IM $(P=0.009)$ [8]. Meanwhile, Coker et al. (2018) found that microbiomes of IM had significantly reduced Chao1-estimated microbial richness compared with that of superficial gastritis. However, there was no significant difference among superficial gastritis, atrophic gastritis and IM based on the evaluation of the overall differences in $\beta$-diversity [11]. In the current study, we found that the diversity of HC-D was higher than that of IM-D based on the Shannon and Simpson indices, while the gastric mucosal microbiota structure of patients with IM differed from that of $\mathrm{HC}$ either with or without $H$. pylori infection, respectively (Fig. 2). The contradiction may be partially caused by different variables which could affect the gut microbiome composition including gender, age, diet and $H$. pylori infection. Further studies focusing on the distribution of gastric microbiota in the development of GC are still needed.

The duodenal mucosal microbiota has garnered considerable attention recently. It has been reported that small intestinal microbiota dysbiosis with an abundance of Proteobacteria influence celiac disease pathogenesis [17]. Li et al. (2017) found that the mucosal microbiota of duodenal samples differed from that of rectal samples in $\mathrm{HC}$; additionally, this difference has been found to be less pronounced in IBS-D. Concurrently, the number of shared OTUs and genera of duodenal rectal samples in IBS-D was more than those of HC. These authors suggested that the shared mucosal-associated microbiota of duodenum and rectum may contribute to the etiology and pathophysiology of IBS-D [8]. It has also been found that the duodenal microbiota of obese individuals displays an alteration in fatty acid and sucrose breakdown pathways possibly induced by diet imbalance [18]. In symptomatic gastritis patients, the patient appraisal of gastrointestinal disorders symptom severity index demonstrated a stronger relation with the duodenal microbiota than with the gastric microbiota. Meanwhile, the combined inflammation score was inversely related with the abundances of $S$. epidermidis $(\mathrm{r}=0.346)$ and $M$. osloensis $(r=0.305)$ in the duodenum [19]. These results indicated that the small intestinal microbiota is an important modulator of health. In this study, we found that the diversity and structure of duodenal mucosal microbiota of patients with IM were significantly different from those of $\mathrm{HC}$ (Fig. 1 and Fig. 2). The results also demonstrate that the HP infection in gastric mucosa had no influence on the structure of the duodenal microbiota (Fig. 2b). Additionally, there were no significant difference between IMG (HP-) and IMD (ANOSIM, R $=0.37$, $P=0.05)$, as well as between HC-G (HP-) and HC-D (ANOSIM, $\mathrm{R}=0.176, P=0.075$ ). Although the sample size was small, these results still suggest that the duodenal microbiota might play a potential role in the pathogenesis of IM, especially in HP negative patients. In future, larger, multicenter studies are needed to explore the role of duodenal microbiota in IM pathogenesis in the future.

As duodenal microbiota dysbiosis was found only in patients with IM, it might be a target for treatment of IM. In recent year, the probiotics have been used to treat many diseases based on modulation the gut microbiota [19-21]. The concentration of living bacteria in commercial probiotics products is much higher as than in the duodenal flora $\left(10^{9}\right.$ vs $10^{5}$ microbes $/ \mathrm{mL}$, respectively). Consequently, probiotic intake may have a greater influence on the duodenal microbiota than on the distal gut microbiota [22]. Probiotics have also been used for treatment of small intestinal bacterial overgrowth in children [23]. Further studies on modulation of the duodenal microbiota for IM treatment through microbiotamodulating therapies, such as probiotics, are needed.

\section{Conclusion}

Duodenal microbiota dysbiosis was found in patients with IM. This dysbiosis might play a role in the pathogenesis of IM and serve as a potential therapeutic target for the condition.

\section{Methods}

\section{Study population}

Patients scheduled for gastroscopy examination were enrolled in this study at Qilu Hospital, Shandong 
University, according to the inclusion and exclusion criteria. The inclusion criteria were as follows: (i) dyspeptic symptoms and older than 40 years; (ii) $H$. pylori infection, IM or AG verified by histological results. The exclusion criteria were as follows: (i) presence of gastrectomy, acute gastrointestinal bleeding, or gastric neoplasia; (ii) presence of conditions unsuitable for the performance of a gastroscopy, such as coagulopathy, impaired renal function (creatinine level $>1.2 \mathrm{mg} / \mathrm{dL}$ ), breastfeeding or pregnancy; (iii) people who did not provide informed consent. In addition, 10 healthy volunteers were examined to ensure that they had no gastritis, metabolic, cardiovascular or cerebrovascular diseases, or cancer and selected as the control group. All volunteers enrolled in this study were not administered pharmacological agents (such as antibiotics, laxatives, antidiarrheal agents, and even antidepressants) or probiotic supplements for at least four weeks prior to the study. This study was approved by the Clinical Ethics Committee of Shandong University Qilu Hospital. All patients and HC received information concerning their participation in the study and provided written informed consent.

\section{Mucosal sample collection, DNA extraction, and pyrosequencing}

The duodenal and gastric mucosal biopsy specimens of patients with IM and $\mathrm{HC}$ were collected. The samples were immediately stored at $-80^{\circ} \mathrm{C}$ and then shipped to Majorbio (Shanghai, China) for high throughput sequencing. FastDNA SPIN kit (MP Biomedicals, California, USA) was used to extract DNA. PCR (ABI GeneAmp 9700, ABI, USA) amplified the V3-V4 region of the bacterial 16S rRNA gene using the primers 338F (ACTCCT ACGGGAGGCAGCAG), and 806R(ACTCCTACGGGA GGCAGCAG), and the TransStartFastPfu DNA Polymerase (TransGen, Beijing, China). Next, the amplicons were purified using gel extraction (AxyPrep DNA GelExtraction Kit, Axygen, California, USA) and quantified using QuantiFluor-ST (Promega, USA). The purified products were pooled to an equimolar concentration, and sequenced using an Illumina MiSeqsystem (Illumina, California, USA) according to standard protocols.

\section{Taxonomy quantification using 16S rRNA gene sequences}

Raw FASTQ data were demultiplexed and qualityfiltered using Trimmomatic and then merged using FLASH according to the following criteria: (i) all reads were deleted at any site achieving an average quality score less than 20 over a 50-bp sliding window. (ii) primers were accurately matched permitting two nucleotide mismatching, and all the reads containing ambiguous bases were eliminated. (iii) Sequences whose overlap was longer than 10-bp were merged by the overlap sequence.
The data analysis was performed on the open cloud platform of Majorbio (www.i-sanger.com). The operational taxonomic units (OTUs) were clustered with the similarity cutoff of $97 \%$ by UPARSE (version 7.1, http:// drive5.com/uparse/), while chimeric sequences were detected and eliminated by UCHIME. The taxonomy of each $16 \mathrm{~S}$ rRNA gene sequence was analyzed by the RDP Classifier algorithm compared to the Silva (SSU128) 16S rRNA database with a confidence threshold of $70 \%$ (http://rdp.cme.msu.edu/).

The abundance of OTUs was normalized by a standard of sequence number according to the least sequences of the samples. Subsequent analysis of $\alpha$ diversity and $\beta$-diversity, principal coordinate analysis (PCoA), were executed by QIIME with these output normalized data. Linear discriminant analysis (LDA) effect size (LEfSe) analyses were executed using the LEfSe tool. Analysis of similarity test (ANOSIM) was carried out with PRIMER 6 software package (PRIMER-E Ltd., Luton, UK) to compare the differences of microbial communities between the patients with IM and HC.

\section{Statistic analyses}

Data are presented as the mean \pm SD. The normality of the distribution was contrasted demonstrated with the Kolmogorov-Smirnov test for normality. The Chi-square test was performed to evaluate the effects of gender. Continuous variables were compared with independent sample and unpaired-samples t-tests. $P$ values $<0.05$ were considered as statistically significant. Analyses were carried out using the SPSS statistical package, version 24.0 (SPSS).

\section{Supplementary information}

Supplementary information accompanies this paper at https://doi.org/10. 1186/s12866-019-1666-5.

Additional file 1: Table S1. The Hp status and a-diversity of each sample.

Abbreviations

GC: Gastric cancer; HC: Healthy control; IM: Intestinal metaplasia; OTU: Operational taxonomic unit

Acknowledgements

Not applicable.

Competing interest

All authors have no conflict of interest related to this study.

Authors' contributions

All authors participated in the conception and design of the study; conceived and drafted the manuscript: JG and YQL; performed the experiments: JG;

collected the basic patient information, clinical indicators: JG; analyzed the data: JG and LXL; revised the paper: XLZ and YQL. All authors read and approved the final manuscript.

\section{Funding}

This study was supported by the National Natural Science Foundation of China (81670489) and Key Research and Development Program of Shandong 
Province (2017CXGC1215). This study is also supported by the Taishan Scholars Program of Shandong Province and National Clinical Research Center for Digestive Diseases supporting technology project (2015BAl13B07). Funding bodies had no role in study design, collected data, analysis, or writing.

\section{Availability of data and materials}

The raw data are available from the SRA database (SRP224905).

\section{Ethics approval and consent to participate}

The protocol of this study was approved by the local Ethical Committee of the Qilu Hospital of Shandong University (NCT02428426). All volunteers received information concerning their participation in the study and gave written informed consent.

\section{Consent for publication}

Not applicable.

\section{Author details}

'Department of Gastroenterology, Qilu Hospital, Shandong University, Jinan 250012, China. ${ }^{2}$ Department of Gastroenterology, Taian City Central Hospital, Taian, Shandong Province, China. ${ }^{3}$ Laboratory of Translational Gastroenterology, Qilu Hospital, Shandong University, No. 107, Wenhuaxi Road, Jinan 250012, China.

Received: 3 July 2019 Accepted: 28 November 2019

Published online: 09 December 2019

\section{References}

1. Siegel RL, Miller KD, Jemal A. Cancer statistics, 2016. CA Cancer J Clin. 2016; 66(1):7-30.

2. Correa P. Human gastric carcinogenesis: a multistep and multifactorial process-first American Cancer Society award lecture on Cancer epidemiology and prevention. Cancer Res. 1992;52(24):6735-40.

3. Correa P. The biological model of gastric carcinogenesis. IARC Sci Publ. 2004:157:301-10.

4. Walker MM. Is intestinal metaplasia of the stomach reversible? Gut. 2003; 52(1):1-4.

5. Malfertheiner P, Megraud F, O'Morain CA, Atherton J, Axon AT, Bazzoli F, Gensini GF, Gisbert JP, Graham DY, Rokkas T, et al. Management of Helicobacter pylori infection--the Maastricht IV/ Florence consensus report. Gut. 2012;61(5):646-64

6. Peek RM Jr, Crabtree JE. Helicobacter infection and gastric neoplasia. J Pathol. 2006;208(2):233-48.

7. Hsieh YY, Tung SY, Pan HY, Yen CW, Xu HW, Lin YJ, Deng YF, Hsu WT, Wu CS, Li C. Increased abundance of Clostridium and Fusobacterium in gastric microbiota of patients with gastric Cancer in Taiwan. Sci Rep. 2018:8(1):158.

8. Li TH, Qin Y, Sham PC, Lau KS, Chu KM, Leung WK. Alterations in gastric microbiota after H. pylori Eradication and in Different Histological Stages of Gastric Carcinogenesis. Sci Rep. 2017;7:44935.

9. Eun CS, Kim BK, Han DS, Kim SY, Kim KM, Choi BY, Song KS, Kim YS, Kim JF. Differences in gastric mucosal microbiota profiling in patients with chronic gastritis, intestinal metaplasia, and gastric cancer using pyrosequencing methods. Helicobacter. 2014;19(6):407-16.

10. Aviles-Jimenez F, Vazquez-Jimenez F, Medrano-Guzman R, Mantilla A, Torres J. Stomach microbiota composition varies between patients with nonatrophic gastritis and patients with intestinal type of gastric cancer. Sci Rep. 2014;4:4202.

11. Coker OO, Dai Z, Nie Y, Zhao G, Cao L, Nakatsu G, Wu WK, Wong SH, Chen Z, Sung JJY, et al. Mucosal microbiome dysbiosis in gastric carcinogenesis. Gut. 2018;67(6):1024-32.

12. Moran C, Sheehan D, Shanahan F. The small bowel microbiota. Curr Opin Gastroenterol. 2015;31(2):130-6.

13. Wacklin P, Laurikka P, Lindfors K, Collin P, Salmi T, Lahdeaho ML, Saavalainen P, Maki M, Matto J, Kurppa K, et al. Altered duodenal microbiota composition in celiac disease patients suffering from persistent symptoms on a long-term gluten-free diet. Am J Gastroenterol. 2014;109(12):1933-41.

14. Chen Y, Ji F, Guo J, Shi D, Fang D, Li L. Dysbiosis of small intestinal microbiota in liver cirrhosis and its association with etiology. Sci Rep. 2016;6: 34055.
15. Pellegrini S, Sordi V, Bolla AM, Saita D, Ferrarese R, Canducci F, Clementi M, Invernizzi F, Mariani A, Bonfanti R, et al. Duodenal mucosa of patients with type 1 diabetes shows distinctive inflammatory profile and microbiota. J Clin Endocrinol Metab. 2017;102(5):1468-77.

16. Li G, Yang M, Jin Y, Li Y, Qian W, Xiong H, Song J, Hou X. Involvement of shared mucosal-associated microbiota in the duodenum and rectum in diarrhea-predominant irritable bowel syndrome. J Gastroenterol Hepatol. 2018;33(6):1220-6.

17. Caminero A, McCarville JL, Galipeau HJ, Deraison C, Bernier SP, Constante M, Rolland C, Meisel M, Murray JA, Yu XB, et al. Duodenal bacterial proteolytic activity determines sensitivity to dietary antigen through protease-activated receptor-2. Nat Commun. 2019;10(1):1198.

18. Angelakis E, Armougom F, Carriere F, Bachar D, Laugier R, Lagier JC, Robert C, Michelle C, Henrissat B, Raoult D. A metagenomic investigation of the duodenal microbiota reveals links with obesity. PLoS One. 2015;10(9): e0137784.

19. Sun $Y Y$, Li M, Li YY, Li LX, Zhai WZ, Wang P, Yang XX, Gu X, Song L, Li Z, Zuo XL, Li YQ. The effect of Clostridium butyricum on symptoms and fecal microbiota in diarrhea-dominant irritable bowel syndrome: a randomized, double-blind, placebo-controlled trial. Sci Rep. 2018;8(1):2964.

20. Martoni CJ, Evans M, Chow CT, Chan LS, Leyer G. Impact of a probiotic product on bowel habits and microbial profile in participants with functional constipation: a randomized controlled trial. J Dig Dis. 2019;20(9): $435-46$.

21. Suk KT, Kim DJ. Gut microbiota: novel therapeutic target for nonalcoholic fatty liver disease. Expert Rev Gastroenterol Hepatol. 2019:13(3):193-204.

22. Han HS, Lee SY, Oh SY, Moon HW, Cho H, Kim JH. Correlations of the Gastric and Duodenal Microbiota with Histological, Endoscopic, and Symptomatic Gastritis. J Clin Med. 2019:8(3).

23. Avelar Rodriguez D, Ryan PM, Toro Monjaraz EM, Ramirez Mayans JA, Quigley EM. Small intestinal bacterial overgrowth in children: a state-of-theart review. Front Pediatr. 2019;7:363.

\section{Publisher's Note}

Springer Nature remains neutral with regard to jurisdictional claims in published maps and institutional affiliations.

Ready to submit your research? Choose BMC and benefit from:

- fast, convenient online submission

- thorough peer review by experienced researchers in your field

- rapid publication on acceptance

- support for research data, including large and complex data types

- gold Open Access which fosters wider collaboration and increased citations

- maximum visibility for your research: over $100 \mathrm{M}$ website views per year

At BMC, research is always in progress.

Learn more biomedcentral.com/submissions 